\title{
Hamstring Strain Injury-Testing and Occurrence Prediction
}

\author{
Łukasz Oleksy ${ }^{1,2}$, Anna Mika $^{1^{\star}}$, Renata Kielnar ${ }^{3}$ and Wojciech Czarny ${ }^{4}$ \\ ${ }^{1}$ Department of Clinical Rehabilitation, University of Physical Education in Krakow, Poland \\ ${ }^{2}$ Oleksy Physiotherapy Clinic, Poland \\ ${ }^{3}$ Faculty of Medicine, University of Rzeszów, Poland \\ ${ }^{4}$ Department of Physical Education, University of Rzeszów, Poland \\ "Corresponding author: Anna Mika, Department of Clinical Rehabilitation, University of Physical Education in Krakow, Poland, Tel: 48126831134; E- \\ mail: anna.mika@awf.krakow.pl
}

Received date: January 04, 2018; Accepted date: January 15, 2018; Published date: January 18, 2018

Copyright: () 2018 Oleksy L, et al.This is an open access article distributed under the terms of the Creative Commons Attribution License, which permits unrestricted use, distribution, and reproduction in any medium, provided the original author and source are credited

\section{Editorial}

A hamstring strain injury [HSI] is the most common noncontact injury in elite sport $[1,2]$. HSI represent $14 \%$ of all injuries in elite soccer accounting for $37 \%$ of all muscle injuries in professional sport [3]. The recurrence rate of HSI ranging from $16 \%$ to $60 \%$ across different sports discipline [2,4]. Risk factors for HSI include age, previous injuries, and changes in muscles strength, flexibility and fatigue [1]. A meta-analysis by Freckleton et al. identified age, previous hamstring injury and increased quadriceps peak torque as most important risk factors. However, the hamstring peak torque, hamstrings-to-quadriceps ratio, and eccentric hamstring strength were not associated with an increased risk of primary or recurrent HSI. But, they underlined that results from different studies are difficult to conclude, because of methodological differences [5]. The biomechanics of hamstring activity during running was evaluated by some authors [6,7]. They have found that the hamstrings are active from mid-swing to terminal stance [7-10]. Hamstrings contract eccentrically during running or kicking a ball, when they slow the forward swing of the leg to prevent overextension of the knee and flexion of the hip [11]. During those movements eccentric hamstring contractions produce strains, and probably microscopic damage to muscle fibers. It was suggested that those microscopic areas of damage may provide a point of weakness resulting a major tear [11-13].

But the major limitation of the studies investigating the hamstring biomechanics is that they generally do not differentiate between the behaviour of the muscle contractile element and other elastic tissues [14]. Studies have shown that the muscle contractile element and elastic tissues may behave differentially and therefore, lengthening of the motor unit does not necessarily indicate lengthening of the muscle contractile element $[15,16]$. Van Hooren et al. raised an important question, if during swing phase hamstrings actions are eccentric, concentric or isometric? They have suggested that elastic tissues could lengthen while the contractile element remains isometric, so increasing distance between the attachment points cannot simply be interpreted as an eccentric muscle contractile element action [14]. Isokinetic muscle strength assessment is a popular screening tool for identifying athletes at risk of future HSI [17]. Some studies found that isokinetic assessment is not predictive for HSI [18], while other studies found its predictive ability [19]. Some authors have suggested that hamstring strains were significantly associated with a low hamstrings-toquadriceps ratio of peak torque on the injured side and a low hamstrings side-to-side ratio of peak torque [11]. But other studies have reported that hamstring strains are not related to a low hamstrings- to-quadriceps strength ratio [18] or they reported normal strength after injury [11]. Green et al. in meta-analysis have reported a week validity of isokinetic testing in hamstring injury prediction [17]. Specifically they underlined, that wide range of measurement protocols was used across the studies (concentric or eccentric isokinetic knee flexor, knee extensor and hip extensor strength were examined at angular velocities ranging $30-300 \%$ s), therefore the results are difficult to interpret unambiguously. Based on their meta-analysis they have reported only a small predictive effect for eccentric knee flexor strength measured at $60 \%$ s angular velocity. No other testing speed or strength ratio showed any association with future hamstring injury. But, they have suggested that the mechanism of hamstrings injury is more complicated and more research is needed in this area.

Van Dyk et al. examined whether differences in isokinetic strength measures of knee flexion and extension represent risk factors for hamstring injuries in professional soccer players [20]. They found small associations between lower hamstring eccentric strength and lower quadriceps concentric strength with hamstrings strain injury and concluded, that the use of isokinetic testing to determine the association between strength differences and HSI should be considered with caution [1,20]. Moreover they underlined that the small association between strength measurements and risk factors highlights the multifactorial nature of hamstring injury. The important aspect of isokinetic testing is the aim of this measurement. The standard protocol may be appropriate when we need to identify athletes with strength imbalances. It was indicated, that a concentric and eccentric isokinetic assessment may be useful as a preseason screening tool for early detection of strength imbalances, what is linked with hamstring injury [21]. It was reported that sprinters who were injured have weakness of the injured limb compared to the uninjured limb, specifically for the knee flexors eccentrically and the hip extensors concentrically, when tested with an isokinetic device at angular velocity $60 \%$ [22]. The important limitation of knee flexor and extensor muscles isokinetic evaluation is the standard seated position of the subject. This position with the hip joint flexed to $90^{\circ}$ and full knee extension during measurement does not allow the knee flexors achieve the maximal range of motion and does not stretch the hamstring muscle group. Therefore it was suggested by Tyler et al. that hamstrings should be tested in stretched position, when function is assessed close to the true end of range of motion [23]. The other important issue is the accurate correction of joint torques and passive muscle tension during isokinetic testing. The gravity correction procedure due to limb mass involves a single measure of torque with the limb relaxed at a specific angle. It is typically performed in standard sitting position. But, when the hamstrings are in lengthen position it was postulated that more than $50 \%$ of the measured torque 
may be due to the combination of limb mass and passive tension [23] It may be the crucial confounding factor in isokinetic contractile torque measurement. The authors have suggested that this problem may be solved using isometric testing; therefore isometric testing is preferable to isokinetic [23].

Considering that hamstring strains often occur in positions of significant stretch, it was suggested that during rehabilitation it is important to provide eccentric strengthening with the hamstrings in a maximally stretched position [6]. Failing to increase an athlete's eccentric strength in a lengthened position after a hamstring injury may predispose an athlete to subsequent reinjury [24]. This approach was confirmed by Tyler et al. study where forty-eight athletes with unilateral hamstring strains followed a 3-phase rehabilitation protocol emphasising eccentric strength training with the hamstrings in a stretched position [25]. Prior to return to sport, isometric strength was assessed bilaterally at $80^{\circ}, 60^{\circ}, 40^{\circ}$ and $20^{\circ}$ knee flexion in sitting with the thigh flexed to $40^{\circ}$ above horizontal. They concluded that rehabilitation with an emphasis on eccentric strength training with the hamstrings in a stretched position resulted in zero recurrent injuries at 2 years after return to play. Athletes who did not perform lengthenedstate eccentric training had a high $50 \%$ recurrence rate $[23,25]$. Additionally Van Hooren et al. have suggested that because during the swing phase of running the action of hamstring contractile element is no eccentric, but rather predominantly an isometric, the isometric rather than eccentric hamstrings exercises are more appropriate and specific for runners [26]. The appropriate hamstrings evaluation protocols should be used for injury screening, managing rehabilitation and determining readiness for return to play. It appears that the standard isokinetic testing-concentric or eccentric may be useful for muscles weakness screening and for the evaluation of training effectiveness. The week association between isokinetic measurements and hamstring strain injury prediction allow suggesting that this option of assessment is not recommended. Based on data from the current literature it appear that the best mode of hamstrings evaluation in the context of injury prevention is the isometric bilateral flexion torque symmetry measurement when the hamstrings are in lengthened, fixed position. When deficits are within $10 \%$, predisposition to reinjury is significantly reduced [24,27].

\section{References}

1. van Dyk N, Bahr R, Whiteley R, Tol JL, Kumar BD, et al. (2016) Hamstring and quadriceps isokinetic strength deficits are weak risk factors for hamstring strain injuries: A 4-year cohort study. Am J Sports Med 44: 1789-1795.

2. Walden M (2005) UEFA champions league study: A prospective study of injuries in professional football during the 2001-2002 season. Br J Sports Med 39: 542-546.

3. Woods C (2004) The football association medical research programme: An audit of injuries in professional football. Analysis of hamstring injuries. Br J Sports Med 38: 36-41.

4. Ekstrand J, Hagglund M, Walden M (2011) Injury incidence and injury patterns in professional football: The UEFA injury study. Br J Sports Med 45: 553-558.

5. Freckleton G, Pizzari T (2013) Risk factors for hamstring muscle strain injury in sport: A systematic review and meta-analysis. Br J Sports Med 47: 351-358.

6. Schache AG, Dorn TW, Blanch PD, Brown NA, Pandy MG (2012) Mechanics of the human hamstring muscles during sprinting. Med Sci Sports Exerc 44: 647-658.
7. Chumanov ES, Heiderscheit BC, Thelen DG (2011) Hamstring musculotendon dynamics during stance and swing phases of high-speed running. Med Sci Sports Exerc 43: 525-532.

8. Kyrolainen H, Avela J, Komi PV (2005) Changes in muscle activity with increasing running speed. J Sports Sci 23: 1101-1109.

9. Kyrolainen H, Komi PV, Belli A (1999) Changes in muscle activity patterns and kinetics with increasing running speed. J Strength Cond Res 13: 400-406.

10. Yu B, Queen RM, Abbey AN, Liu Y, Moorman CT, et al. (2008) Hamstring muscle kinematics and activation during overground sprinting. J Biomech 41: 3121-3126.

11. Brockett CL, Morgan DL, Proske U (2004) Predicting hamstring strain injury in elite athletes. Med Sci Sports Exerc 36: 379-387.

12. Garrett WE (1990) Muscle strain injuries: Clinical and basic aspects. Med Sci Sports Exerc 22: 436-443.

13. Brockett CL, Morgan DL, Proske U (2001) Human hamstring muscles adapt to eccentric exercise by changing optimum length. Med Sci Sports Exerc 33: 783-790.

14. Van Hooren B, Bosch F (2017) Is there really an eccentric action of the hamstrings during the swing phase of high-speed running? part 1: A critical review of the literature. J Sports Sci 35: 2313-2321.

15. Finni T, Komi PV, Lepola V (2000) In vivo human triceps surae and quadriceps femoris muscle function in a squat jump and counter movement jump. Eur J Appl Physiol 83: 416-426.

16. Kurokawa S, Fukunaga T, Nagano A, Fukashiro S (2003) Interaction between fascicles and tendinous structures during counter movement jumping investigated in vivo. J Appl Physiol 95: 2306-2314.

17. Green B, Bourne MN, Pizzari T (2017) Isokinetic strength assessment offers limited predictive validity for detecting risk of future hamstring strain in sport: A systematic review and meta-analysis. Br J Sports Med.

18. Bennell K, Wajswelner H, Lew P, Schall-Riaucour A, Leslie S, et al. (1998) Isokinetic strength testing does not predict hamstring injury in Australian Rules footballers. Br J Sports Med 32: 309-314.

19. Fousekis K, Tsepis E, Poulmedis P, Athanasopoulos S, Vagenas G (2011) Intrinsic risk factors of non-contact quadriceps and hamstring strains in soccer: A prospective study of 100 professional players. Br J Sports Med 45: 709-714.

20. van Dyk N, Bahr R, Burnett AF, Whiteley R, Bakken A, et al. (2017) A comprehensive strength testing protocol offers no clinical value in predicting risk of hamstring injury: A prospective cohort study of 413 professional football players. Br J Sports Med 51: 1695-1702.

21. Croisier JL, Ganteaume S, Binet J, Genty M, Ferret JM (2008) Strength imbalances and prevention of hamstring injury in professional soccer players: A prospective study. Am J Sports Med 36: 1469-1475.

22. Sugiura Y, Saito T, Sakuraba K, Sakuma K, Suzuki E (2008) Strength deficits identified with concentric action of the hip extensors and eccentric action of the hamstrings predispose to hamstring injury in elite sprinters. J Orthop Sports Phys Ther 38: 457-464.

23. Tyler TF, Schmitt BM, Nicholas SJ, McHugh MP (2017) Rehabilitation after hamstring-strain injury emphasizing eccentric strengthening at long muscle lengths: Results of long-term follow-up. J Sport Rehabil 26: 131-140.

24. Schmitt B, Tim T, McHugh M (2012) Hamstring injury rehabilitation and prevention of reinjury using lengthened state eccentric training: A new concept. Int J Sports Phys Ther 7: 333-341.

25. Tyler TF, Schmitt B, Gellert JM, McHugh MP (2014) Eccentric strengthening at long muscle lengths reduces hamstring strain recurrences: Results of long term follow-up. Orth J Sport Med 2.

26. Van Hooren B, Bosch F (2017) Is there really an eccentric action of the hamstrings during the swing phase of high-speed running? Part 2: Implications for exercise. J Sports Sci 35: 2322-2333.

27. Tyler TF (2016) Evolution of a successful new hamstring rehabilitation protocol to reduce hamstring injury recurrence. Int J Sports Phys Ther 7: 333-341. 